\title{
Factors Contributing to the Delay in the Initiation of Treatment of Patients Enrolled in the TB DOTS Program in a Highly-Urbanized City in the Philippines
}

\author{
Frances Evangeline S. Vista, ${ }^{1}$ Monaflor Abigail G. Ignacio, ${ }^{1}$ Danica Marie C. Bausa, ${ }^{1}$ \\ Danica Francia D. Lanuza, ${ }^{1}$ Justin Paul M. Mijares, ${ }^{1}$ Carl Abelardo T. Antonio ${ }^{2}$ \\ ${ }^{1}$ College of Public Health, University of the Philippines Manila \\ ${ }^{2}$ Department of Health Policy and Administration, College of Public Health, University of the Philippines Manila
}

\begin{abstract}
Objective. The main objective of the study is to determine the factors which contribute to the delay in the initiation of treatment of TB patients enrolled in the TB control program in a highly urbanized city in the Philippines.

Methods. A descriptive, cross-sectional study was conducted in 14 health centers in the city; from which the proportion of TB patients who experienced delay in the initiation of treatment $(>1$ day after diagnosis) was obtained through records review. The health system and patient factors which contributed to the patients' delay were determined through face-to-face interviews. The information obtained was analyzed with supplemental information from interviews with selected health workers in the tuberculosis control program of the city.
\end{abstract}

Results. These showed that $20.95 \%$ of the TB patients enrolled in the TB control program of the city experienced delay in the initiation of treatment. They experienced a mean delay of 9.48 days. The health system and patient factors which affected the patients the most were the referral system (24.5\%) and conflict between treatment and work or household responsibilities (22.4\%), respectively.

Conclusion. These existing delays imply the need to implement interventions directed to improving current measures to control TB in the country.

Key Words: time-to-treatment, tuberculosis, pulmonary, directly observed therapy, Philippines

\section{Introduction}

Tuberculosis (TB) is among the leading causes of death globally, as the World Health Organization (WHO) announced that by 2013, 9 million people had already

Corresponding author: Frances Evangeline S. Vista

College of Public Health

University of the Philippines Manila

625 Pedro Gil St., Ermita, Manila 1000 Philippines

Telephone: +632 5232997

Email: chinvista@gmail.com contracted $\mathrm{TB}$ and approximately $15 \%$ or 1.5 million of whom died. ${ }^{1}$ Most cases (95\%) of this disease come from developing countries such as the Philippines, wherein in 2010, TB was the sixth prominent cause of morbidity and mortality. ${ }^{1}$ The growing concern for TB in the Philippines is persistent despite the national coverage and implementation of the WHO-recommended Directly Observed treatment Short-course chemotherapy (DOTS) since 1997 through the National Tuberculosis Control Program (NTP). ${ }^{2}$ The DOTS strategy aims to facilitate compliance to anti-TB medication since treatment is directly observed by a supervising health worker. Once a patient is treated, TB is stopped at the source; transmission of the disease is intercepted; development of multidrug-resistant $\mathrm{TB}$ is prevented; and occurrence of complications, such as relapse and death, are ultimately averted. ${ }^{3,4}$ However, a potential flaw in the NTP is the delay in the initiation of anti-TB treatment, particularly in the stage of the TB care pathway between diagnosis and treatment. It is posited that a delay in the initiation of treatment presents an opportunity when patients may potentially be lost to the system. This issue is of greater significance for individuals who are known to be highly infectious, i.e. with positive sputum smear microscopy results, as delays may cause increased disease transmission. An untreated smear-positive patient can infect, on average, 10 contacts annually and over 20 individuals during the natural course of the disease until death. ${ }^{5}$ In a study in one of Europe's vital ports and country's second largest city, Rotterdam City, it was revealed that the transmission of the disease in urban areas was found to be 3.8 times higher than semi-urban and rural areas. ${ }^{6}$ This highlights the importance of looking at the disease in the context of our country.

There also exists a knowledge gap in the Philippines regarding this topic. Studies in the country exploring delays in the TB care pathway involve only the delay in the diagnosis of patients. These include papers entitled "Health Seeking and Perceived Causes of Tuberculosis among Patients in Manila, Philippines" and "Barriers of Early TB Diagnosis among the Poor in Highly Urbanized Areas in the Philippines", published in 2000 and 2014, respectively. ${ }^{7,8}$ 
This research aims to determine the factors that contribute to the delay in the initiation of treatment of patients enrolled in the TB DOTS program in a highly urbanized city in the Philippines. Specifically, this study aims: (1) to determine the proportion of patients enrolled in the TB DOTS program at the time of data collection who experienced delay in the initiation of their treatment, (2) to determine the duration of the delay in the initiation of treatment among TB DOTS patients who experienced such delay, (3) to describe the perceived health systems factors that contributed to the delay in the initiation of treatment, and (4) to describe the patient factors, either sociodemographic factors or perceived patient factors, that contributed to the delay in the initiation of treatment.

\section{Methods}

\section{Study Design}

The study utilized a descriptive and cross-sectional design.

\section{Definition of Major Study Variables}

1. Delay in the initiation of treatment - the date of the confirmation of diagnosis of tuberculosis is more than one day apart from the date of the initiation of treatment

2. Duration of delay - the time interval between date of the confirmation of diagnosis and the date of initiation of treatment in days

3. Direct Sputum Smear Microscopy (DSSM) Result whether the TB patient is sputum-negative or sputumpositive

4. Health system factors - components of the health system that may either facilitate or deter access to health care such as service delivery, health workforce, medical products (availability of drugs), health financing, and governance.

5. Patient factors - patient-related elements that promote or hinder access to health care

\section{Study Area}

The city was chosen as the site of the study for feasibility concerns since the researchers had identified persons from the said area to coordinate with. Additionally, the health centers in this city also have a functional information system that would make data collection feasible and efficient. All 14 health centers were included in the study. All of these facilities are in compliance with the NTP.

\section{Study Population}

The sample population varied for the four specific objectives of this study. For the first specific objective, the study population was all the PTB patients enrolled in the TB DOTS program at the time of data collection in all 14 health centers. For the second specific objective, the sample population was new sputum-positive patients who experienced delay in their initiation of treatment. For specific objectives three and four, the sample population was those patients who experienced delay in their initiation of treatment and who met the criteria shown in Table 1.

Table 1. Inclusion and Exclusion Criteria for Patient Interviews

\begin{tabular}{ll}
\hline Inclusion Criteria (must meet all) & Exclusion Criteria (at least one) \\
\hline Aged 18-59 & $\begin{array}{l}\text { Enrolled in the TB DOTS program } \\
\text { in the past }\end{array}$ \\
$\begin{array}{l}\text { Enrolled in the TB DOTS program } \\
\text { at the time of data collection }\end{array}$ & $\begin{array}{l}\text { Extrapulmonary TB patients } \\
\text { New case of smear-positive }\end{array}$ \\
pulmonary TB & Case of MDR-TB \\
Goes to the health center regularly & Sputum negative patientsa \\
for treatment & Retreatment case (Category 2)
\end{tabular}

For the third specific objective, an additional sample population was the laboratory aide, nurse, and doctor who were involved with work with TB DOTS in the health center that had the largest proportion of patients who experienced delay.

\section{Sampling Method}

No sampling method was employed for the TB DOTS patients in the different specific objectives since total enumeration was done.

\section{Data Collection and Data Collection Instrument}

\section{Records Review}

To be able to determine the proportion of patients that experienced delay in the initiation of treatment and the duration of that delay, a records review was conducted. A data abstraction form was given to and filled up by the NTP Nurse Coordinator of the city (Annex A). Some information not available from records were asked from each health center. Records review also allowed the researchers to 
determine patients who qualified the inclusion criteria, and not the exclusion criteria for the interviews.

\section{Interviews}

To be able to determine the health system and patient factors that contributed to the delay in the initiation of treatment, interviews with patients were done. The tool for data collection was an interview schedule that was loosely based on a data collection tool by the United States Agency for International Development (USAID) and literature review (Annex I). ${ }^{9}$ It contained questions that seeks sociodemographic information and perceived health system and patient factors that may have contributed to delay in the initiation of treatment. In asking the patients their reason for delay, the pre-determined factors were not enumerated to them, rather, they expected to cite his/her own perceived patient factors or perceived health system factors that contributed to the delay in the initiation of treatment. After they have enumerated their own reasons, other possible reasons that have might been missed by the patient were asked to them.

Key informant interviews were also conducted in the health center with the largest proportion of patients who experienced delay in initiation of treatment. These were conducted as supplements for the third specific objective, which was to describe health system factors that contributed to delay in the initiation of treatment. Questions regarding the health system factors were constructed based on the current health system setup of the NTP which was described thoroughly in the 2013 Manual of Procedures (MOP) for the NTP. ${ }^{10}$

Both interview schedules were pre-tested in a health center in Las Piñas City.

\section{Data Processing and Analysis}

The data abstraction forms from records review were encoded using Microsoft Excel, while the results of the patient interviews were encoded using a form created in Epi Info 3.5.4. The data collected were double-checked and double-encoded for accuracy. The key informant interviews were transcribed and summarized in a narrative report. All data were analyzed using Epi Info 7.

\section{Institutional Review}

The study protocol was reviewed and approved by the PH 197/199 Teaching Committee of the College of Public Health (technical review) and the UP Manila Research Ethics Board Panel 5b (College of Public Health).

\section{Results}

Based on the data abstracted from the NTP records of the city, a total of 420 patients were enrolled in the DOTS program of health centers of the city as of December 31, 2014. Of this number, records of 339 (80.71\%) patients were further reviewed to determine the magnitude of delay in initiation of treatment. Application of inclusion and exclusion criteria identified 49 patients for interview to determine the perceived patient and health system factors affecting delay in initiation of treatment (Figure 1).

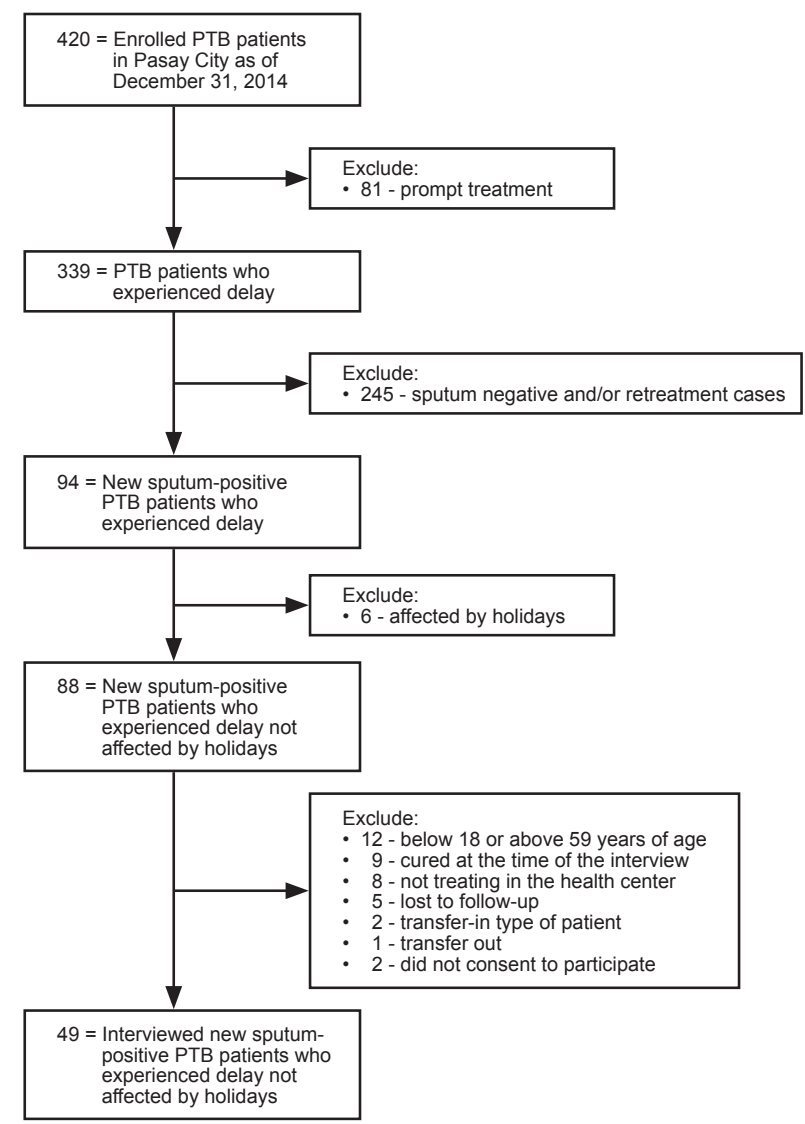

Figure 1. Inclusion and Exclusion Schematic Diagram

\section{Proportion of Patients Who Experienced Delay}

Of the initial 420 PTB patients enrolled, 20.95\% are sputum-positive patients that were treated more than one day after receiving their DSSM result.

\section{Duration of Delay}

The mean duration of delay of new sputum-positive patients is 9.48 days. For other measures of central tendency, the median was found to be 5.00 days while the mode was 2.00 days. For measures of statistical dispersion, the standard deviation was found to be 14.41 days, while the range was 90.00 days.

\section{Sociodemographic Factors}

From the total 49 patients interviewed, most of the patients were found to be males $(75.5 \%)$. Most subjects are found to be part of the 18 -30 age group (38.8\%) For financial characteristic of the interviewed patients, majority came from the lowest quintile, $0-3500$ Philippine pesos (PHP) making 
up almost half of the interviewed subjects (42.9\%). About half (51\%) of those interviewed were high school graduates. A total of $38.8 \%$ of the subjects were unemployed while $34.7 \%$ were contractual workers. For the last socio-demographic information, the number of household members living with the TB patient, $36.7 \%$ answered that they live with a range of 7-9 people under their roof. (Table 2)

Table 2. Socio-demographic Characteristics of Patients who Experienced Delay in Initiation of Treatment, Highly Urbanized City, 2014

\begin{tabular}{|c|c|c|c|}
\hline \multicolumn{2}{|c|}{ Socio-demographic Information } & \multicolumn{2}{|c|}{$\begin{array}{c}\text { Proportion of Patients } \\
\text { (total = 49) }\end{array}$} \\
\hline & & $\mathbf{n}$ & $\%$ \\
\hline \multirow{2}{*}{ Sex } & Female & 12 & 24.5 \\
\hline & Male & 37 & 75.5 \\
\hline \multirow{4}{*}{ Age } & $18-30$ & 19 & 38.8 \\
\hline & $31-40$ & 7 & 14.3 \\
\hline & $41-50$ & 12 & 24.5 \\
\hline & $51-59$ & 11 & 22.4 \\
\hline \multirow{7}{*}{$\begin{array}{l}\text { Monthly } \\
\text { Income }\end{array}$} & $0-3500$ & 21 & 42.9 \\
\hline & $3501-6000$ & 6 & 12.2 \\
\hline & $6001-9501$ & 10 & 20.4 \\
\hline & $9501-38,000$ & 8 & 16.3 \\
\hline & 38,000 and above & 1 & 2.0 \\
\hline & Did not say & 3 & 6.1 \\
\hline & Elementary & 11 & 22.4 \\
\hline Highest & High School & 25 & 51.0 \\
\hline Educational & Vocational Course Graduate & 3 & 6.1 \\
\hline \multirow[t]{2}{*}{ Attainment } & College Undergraduate & 8 & 16.3 \\
\hline & None & 2 & 4.1 \\
\hline \multirow{4}{*}{ Occupation } & Contractual & 17 & 34.7 \\
\hline & Permanent & 5 & 10.2 \\
\hline & Self-employed & 8 & 16.3 \\
\hline & Unemployed & 19 & 38.8 \\
\hline \multirow{4}{*}{$\begin{array}{c}\text { Number of } \\
\text { Household } \\
\text { Members }\end{array}$} & $1-3$ & 6 & 12.2 \\
\hline & $4-6$ & 13 & 26.5 \\
\hline & $7-9$ & 18 & 36.7 \\
\hline & 10 and above & 12 & 24.5 \\
\hline
\end{tabular}

\section{Perceived Patient Factors}

Table 3 shows the perceived patient factors contributing to delay in the initiation of treatment according to the patients. Among these, the most prevalent was the conflict between the schedule of treatment and work or responsibilities at home.

\section{Perceived Health System Factors}

Table 4 shows the perceived health system factors contributing to the delay in initiation of treatment according to patients. The most frequently cited factor by patients was the referral system.

\section{Discussion}

Delays between the time of confirmation of diagnosis and initiation of treatment still exist in the city. The duration of this delay is shown to be longer than in other areas according to various studies. ${ }^{11,12,13}$ This comparison is shown below in Table 5 .
Table 3. Perceived Patient Factors Contributing to Delay in the Initiation of Treatment According to the Patients

\begin{tabular}{lcc}
\hline & \multicolumn{2}{c}{$\begin{array}{c}\text { Proportion } \\
\text { of patients }\end{array}$} \\
\cline { 2 - 3 } & $\mathbf{n}$ & $\mathbf{\%}$ \\
\hline Conflict between work (if employed) or responsibilities at & 11 & 22.4 \\
home (if unemployed) and treatment & 5 & 10.2 \\
Belief that the disease is not severe and fatal & 5 & 10.2 \\
Other appointments & 5 & 10.2 \\
Hindrances due to physical conditions & 3 & 6.1 \\
In denial of having the disease & 3 & 6.1 \\
Lack of will & 2 & 4.1 \\
Financial burden of treatment & 2 & 4.1 \\
Embarrassment due to social stigma associated with TB & 1 & 2.0 \\
Preference for other types of treatment & 1 & 2.0 \\
Vices & 1 & 2.0 \\
Bad weather & 1 & 2.0 \\
Undergoing other diagnostic procedures after Direct & & \\
Sputum Smear Microscopy (DSSM) & &
\end{tabular}

Table 4. Perceived Health System Factors Contributing to Delay in the Initiation of Treatment According to the Patients

\begin{tabular}{lcc}
\hline \multicolumn{1}{c}{ Perceived health system factors } & \multicolumn{2}{c}{$\begin{array}{c}\text { Proportion } \\
\text { of patients* }\end{array}$} \\
\cline { 2 - 3 } & $\mathbf{n}$ & $\%$ \\
Referral system & 12 & 24.5 \\
Advice to return for the result on a date more than 1 day & 6 & 12.2 \\
from the date of diagnosis & 4 & 8.2 \\
Mass vaccination campaign (Operation Ligtas sa Tigdas) & 2 & 4.1 \\
The health workers' (e.g. doctor, nurse) encouragement & 2 & 4.1 \\
to initiate treatment was not enough & 2 & 4.1 \\
Lack of instruction on when to return for the DSSM result & 1 & 2.0 \\
$\begin{array}{l}\text { Different treatment given before official TB medication } \\
\text { Long travel time to the health center }\end{array}$ & & \\
\hline${ }^{*}$ Can have multiple answers &
\end{tabular}

Table 5. Range and Median of the Time Period between the Confirmation of Diagnosis and Initiation of Treatment for All TB Patients in Various Areas and Years

\begin{tabular}{lcc}
\hline Area & Range & Median \\
\hline City X* (all PTB patients) & 111 & 11 \\
City X* (all new sputum-positive patients) & 92 & 4 \\
${\text { Bao' an District, Shenzhen, China }{ }^{11}}^{\text {Beira City, Mozambique }{ }^{12}}$ & 73 & 0 \\
Udupi Taluk, Udupi District, & 3 & 1 \\
Karnataka State, South India ${ }^{13}$ & 2.8 & 2 \\
${ }^{*}$ City X refers to the highly urbanized city in the Philippines being discussed in the \\
study
\end{tabular}

\section{Sociodemographic Factors}

It is essential to describe the sociodemographic factors of the population interviewed because these may help in the understanding of the perceived patient and health system factors that the researchers have found. Some parameters may also be compared to other literature. For instance, it was observed that $75 \%$ of those interviewed were males. In a study done in China that also quantified patients who experienced delay in initiation of treatment, majority $(65 \%)$ were also males. ${ }^{14}$ However, it must be noted that most TB 
patients in the health centers of the city are males in the first place. The researchers also found that most of the patients who experienced delay are from the 18-30 age bracket, which is consistent with WHO's statement that tuberculosis affects the productive age group more than the children and elderly. ${ }^{1}$

\section{Perceived Patient Factors}

Patient factors are those that rest upon the actions and decisions made by individuals. For example, patients may choose to do other tasks, such as visiting family members, or procrastinate getting treatment because of vices. While these patients had other things to do causing their failure to get prompt treatment, some, on the other hand, had the ability to get treated immediately, but had the lack of will to do so. Alibis such as crowding in the health center and taking the close proximity of the health center for granted have been cited for such lack of will. It is also possible that physical incapability to go to the health center was used as an alibi, though the evidence for this is insufficient. This is probable since these patients were able to go to the health center for diagnosis, but suddenly because of perceived incapabilities, he or she could not start treatment immediately. There was also a patient who underwent other diagnostic procedures, particularly a chest x-ray, even after having a positive result in DSSM diagnosis. The patient was not able to report who ordered the chest x-ray, but nevertheless, this caused the delay.

The decision of some interviewed patients to delay initiation of treatment was also based on some notions they may have. There are some who had the belief that the disease is not severe or fatal. Inferences for having such reason are the mild symptoms TB presents with during its early stages. However, some patients deny that they have the disease at all. This has been treated as an ongoing problem, as evident even in the MOP. ${ }^{10}$ Unfortunately, different perceptions may have also come to play, leading to this disbelief. For example, some patients have wrongfully associated TB with genetic diseases which may be passed down from their ascendants. This may have convinced them that treatment contributes less to their wellness. Health workers have also cited that some patients associate their disease with local beliefs such as witchcraft or kulam and other supernatural phenomena. ${ }^{15}$ Some patients were also in denial because of the fear that other people may be disgusted with them. This is related to the social stigma associated with TB that has also been found to be contributory to delay in this study. Since TB is a highly infectious and often fatal disease, many people do not prefer the company of afflicted patients mainly out of fear of infection. ${ }^{16}$ This may include peers and family members as well as present or potential employers. This social phenomenon is still existing in the Philippines. ${ }^{17}$ According to a study in India, social stigma has been a persistent barrier to health-seeking behavior of patients due to the fear of damaging social status, social relations as well as their source of income. ${ }^{18}$ This factor has a negative impact on the health system, contributing to delays and in seeking healthcare services of the different stages in the TB care pathway. ${ }^{8}$ Perceptions not only regarding the disease, but also regarding the TB treatment itself influences patients' behavior to get treatment. This is illustrated by some patients considering other types of treatment other than what is offered by the health center. Filipinos generally associate the private sector with better quality of services, but prefer public services mostly for the cost. ${ }^{19}$ Some responses of the patients in this study attest to that. However, unbeknownst to some of those patients, the services offered by the private and public sector regarding TB treatment are the same, differing only in the cost. This causes the patient to return to get treated at a public health facility, already delayed.

However, not all these factors may be attributed to the patient alone. There are certain elements that may have driven the patient to take the course of action he or she chose to do. For instance, bad weather, specifically typhoons, is an uncontrollable natural calamity that was found to affect delay in initiating TB treatment. Delays caused by the financial burden related to getting treatment cannot be solely attributed to the patient because they did not desire to have problems with expenses. Even though the sputum tests and TB drugs can be acquired for free from the health center, some financial burden still comes with undergoing DOTS such as having to commute to the health center. This economic burden on patients stems from the urban poverty that still continues to plague urbanized areas of the Philippines. ${ }^{19}$ The same can be said about those that had conflict between their work schedule and treatment. For a third world country such as the Philippines, it is not uncommon to find patients who would choose to work first before doing anything else. Patients often state that even though they are aware that they have to prioritize their health, they would have nothing to eat or feed their families if they stop working.

\section{Perceived Health System Factors}

Being referred to DOTS facilities is important since patients enrolled in the TB DOTS program have increased chances of compliance to the treatment regimen. ${ }^{10}$ Thus, the referral system is truly an integral part of the NTP. It is stated in the MOP that the referral system should be as orderly as possible to be able to enforce prompt diagnosis and treatment of TB patients, ${ }^{10}$ however, referral has been cited as the most prevalent factor causing delay. As other studies have identified, the lack of coordination between public providers are causing delay. ${ }^{20}$ The patients who cited this factor as a reason for delay are those that underwent procedures that are not in accordance with the system the NTP has laid out 
because of having prior diagnosis in a facility other than the one wherein they received treatment. These patients, though successfully diagnosed, cannot be treated on the same day or one day after because of having to move from facility to another. It is inevitable that patients preferred to be checked in other health facilities other than health centers because of various reasons such as severity of symptoms, proximity, advice of friends of relatives, working hours of the health center, and availability of the doctor at the health center. The health provider, however, cannot be held fully accountable for delays due to referral. As told by key-informants, some patients in the past that are not part of this study failed to bring necessary documents such as referral slips or old treatment cards. Lacking both or one of these papers, the patients had to undergo a confirmatory sputum examination before being able to initiate treatment. The burden of this particular factor falls on the shoulders of the health care providers since they are the ones who implement these protocols, most of which are not known to the patients.

Accessibility to the DOTS facility also contributes largely to the quality of health service delivery being provided and it is a policy in the MOP to ensure that DOTS be done in a setting which is most accessible to the patient. The accessibility of health centers was generally not found to be a barrier in getting prompt TB treatment since almost all patients have stated that they live near the health center where they are treated in. Additionally, key informants have said that there are health centers close to each barangay in the city. One has even mentioned that the longest travel time to a health center would probably be around 20 minutes of walking. This is compliant with the standard of a 30-minute travel time to a nearest health facility according to Bosanac. ${ }^{21}$ However, there was one patient who stated that long travel time to the health center was the reason for not getting prompt treatment because of the proximity of the health center to his workplace.

Some services from the health center may be individualized for each patient, depending on their needs. In this study, some delays have been found to be a result of being prescribed a different medication before anti-TB treatment based on the physician's own discretion. This is because some patients may harbor co-infections such as pneumonia, and may have been given different treatment such as co-trimoxazole as an empiric treatment while waiting for the result of the DSSM. Some may need management for symptoms such as fever and coughing. Patients were allowed to finish their empiric treatment first before shifting to the TB treatment. These departures from the existing MOP may cause delay in the initiation of treatment of patients. However, these kind of delays are sometimes unavoidable since these decisions would be for the betterment of the patients' health.

Among the cited factors, the lack of encouragement to start treatment is related to this component of the health system. The first patient who cited this factor claimed not being informed of having tuberculosis. This claim, however, could be due to other factors as well, such as the level of understanding of the patient. The other patient who cited this, on the other hand, was allowed to attend to another medical checkup for work before initiating treatment immediately. These situations are sometimes unavoidable and cannot be fully attributed to healthcare workers. The general finding in this study, in contrast to these two cases, is that the city generally has an effective human health resource. It was explained by key informants that a lab aide or nurse is readily available in the health centers of the city to administer directly-observed treatment to patients. There has been good feedback from a number of patients about how persistent the health care personnel are in urging them to start and be compliant with treatment. Additionally, according to key informants, proper counselling to TB patients is done by health workers. This is in line with what is written in the MOP which is to effectively impart TB communication messages to patients and conduct health education sessions. ${ }^{10}$

It was found that in some health centers, patients were advised to return after around three days for their DSSM results. It is strongly speculated that this is due to the protocol written in the MOP for the NTP that results of DSSM must be sent back to the requesting unit within three working days from the time the sputum was obtained. Nevertheless, the researchers also found out that at times, the results have been available on the same day the sputum was obtained, but patients are not treated immediately because they have been advised in advance to return after three days. However, some were not advised to return at all. The patient then took it upon themselves to come back anyway, thus causing the delay. This problem arises when there is misinformation or lack of information given to the patients.

In addition to this, there were also situations where patient's initiation of treatment was rescheduled for another month due to the Operation Ligtas sa Tigdas. This points back to the health service delivery of the center as well. Health facilities must be efficient and effective enough to manage the different operations and programs they have to control a variety of diseases. An outbreak of one disease must not be a reason for halting all other services they have for other diseases. It is possible that the health centers were aware of the negative consequences this may have on other patients such as TB patients, but they were not able to propose an alternative because of lack of staff. Even if they had enough members of the TB task force, BHWs, lab aides, nurses, and physicians that work to deliver health services that the NTP promises, this apparently appeared to be not enough for emergency cases such as this.

The researchers have uncovered that some delays according to the definition in this study, were due to 
inconsistencies in the recording system. Some patients who've undergone the referral system, the dates of their diagnosis are from their original health facilities, while the dates of their initiation of treatment are the dates of transfer to their respective health centers in the city, and not the day when they really started taking anti-TB medication. Records review further revealed that some patients had dates of initiation of treatment that came before the date of their DSSM result. Upon inquiry with the NTP nurse coordinator, it was explained that this was because these patients started treating at a private facility, and they decided to transfer to the health center, probably because medication is free. DSSM had to be repeated in the health center for these patients, probably because the private facilities are not DOTScertified. Thus, their DSSM result on their record is actually the date when they were transferred.

\section{Synthesis}

Though presented separately, the health system and patient factors are not isolated from each other. An example, is the blaring connection between factors is the relation between travel time to the health facility which is a health system factor, and patient factors related to financial burden and prioritizing work and other responsibilities. Some patients experienced delay because of the health centers' "turfing" - meaning patients are restricted to health centers within the jurisdiction of their place of residence. Even when in fact their workplace is closer to health centers in other cities, they are assigned to health centers in cities where they live, causing financial burden and long travel times because of commuting. The same thing could be said for the problem about the referral system that patients bring incomplete documents or need to repeat sputum exams, causing delay.

While being rigid in some aspects, the facilities are also sometimes flexible with some protocols of the MOP. Some patients experienced delay in receiving their anti-TB drugs because they were given other medication or were advised to first finish the batch of antibiotics that they were talking at the time of diagnosis. The health workers advise this on some cases even though it is clearly stated in the MOP that once diagnosis has been confirmed, treatment should be started right away. This just shows the need of the facilities to reassess their compliance to the MOP and adjust so that the patience may receive the optimum care that the facilities can offer.

\section{Conclusion}

Among all enrolled TB patients in the health centers, $20.95 \%$ are new sputum-positive patients who experienced delay in initiation of treatment. This shows that although prompt treatment is advocated by the MOP for the NTP, delays in initiation of treatment, especially among new sputum positive TB patients, still persist in the city. The average duration of this delay is 9.48 days, during which the disease can be continually transmitted. Furthermore, the duration of the delay in the initiation of treatment may result to the health system, in particular the NTP, losing these patients. These present the cause of concern for the public health sector.

Both health system and patient factors were found to contribute to the delay of initiation of treatment. The most prevalent health system factor is the referral system $(24.5 \%)$, while the conflict between treatment and work or household responsibilities is the most prevalent patient factor $(22.4 \%)$. Aside from these, six other health system factors and 11 other patient factors have been uncovered. This implies the need to implement interventions directed to both individuals diagnosed with TB and the NTP to further improve the current measures directed to reduction of the prevalence of TB in the country.

\section{References}

1. World Health Organization. Global tuberculosis report. Geneva: World Health Organization Press; 2014. p. 13.

2. Department of Health. 2010-2016 Philippine plan of action to control tuberculosis (PhilPACT). Manila: Department of Health; 2010. p. 21.

3. Department of Health. Manual of operations for the national TB control program. Manila: Department of Health; 2005.

4. World Health Organization. An expanded DOTS framework for effective tuberculosis control. Geneva: World Health Organization; 2002. pp. 5-7.

5. World Health Organization. Diagnostic and treatment delay in tuberculosis. Cairo: World Health Organization Regional Office for the Eastern Mediterranean; 2006. p. 11.

6. de Vries G, Van Hest NA, Baars HW, Sebek MM, Richardus JH. Factors associated with the high tuberculosis case rate in an urban area. Int J Tuberc Lung Dis. 2010; 14(7):859-65.

7. Auer C, Sarol J Jr, Tanner M, Weiss M. Health seeking and perceived causes of tuberculosis among patients in Manila, Philippines. Trop Med Int Health. 2000; 5(9):648-56.

8. Reyes K, Amores J. Barriers of early TB diagnosis among the poor in highly urbanized areas in the Philippines. Philippine Institute for Development Studies. 2014; 18(11):16-17.

9. United States Agency International Development. Reducing delays in TB diagnosis data collection tools to evaluate the cause and frequency of TB delays. 2011. pp. 3-28.

10. Department of Health. Manual of procedures. Manila: Department of Health; 2013. pp. 22-105.

11. Xu X, Liu JH, Cao SY, et al. Delays in care seeking, diagnosis and treatment among pulmonary tuberculosis patients in Shenzhen, China. Int J Tuberc Lung Dis. 2013; 17(5):615-20.

12. Saifodine A, Gudo PS, Sidat M, Black J. Patient and health system delay among patients with pulmonary tuberculosis in Beira city, Mozambique. BMC Public Health. 2013; 13:559.

13. Goel K, Kondagunta N, Soans SJ, Bairy AR, Goel P. Reasons for patient delays and health system delays for tuberculosis in South India. Indian J Community Health. 2011; 23(2):87-9.

14. Zhou C, Chu J, Geng H, Wang X, Xu L. Pulmonary tuberculosis among migrants in Shandong, China: factors associated with treatment delay. BMJ Open. 2014; 4(12):e005805. 
15. Abad P, Tan M, Baluyot M, et al. Cultural beliefs on disease causation in the Philippines: challenge and implications in genetic counseling. J Community Genet. 2014; 5(4):399-407.

16. Courtwright A, Turner AN. Tuberculosis and stigmatization: pathways and interventions. Public Health Rep. 2010; 125 Suppl 4:34-42.

17. Alterado A. Stigma and having tuberculosis: Agenda for research. Asia Pacific E-Journal of Health Social Science. 2013; 2(1):1-4.

18. Atre S, Kudale A, Morankar S, Gosoniu D, Weiss MG. Gender and community views of stigma and tuberculosis in rural Maharashtra, India. Glob Public Health 2011; 6(1):56-71.

19. Department of Health. National objectives for health Philippines, 2011-2016. Manila: Health Policy Development and Planning Bureau (HPDPB) Department of Health; 2012. pp. 3-17.
20. World Health Organization. Everybody business: strengthening health systems to improve health outcomes: WHO's framework for action. Geneva: World Health Organization; 2007. pp. 14-25.

21. Finnie RK, Khoza LB, van den Borne B, Mabunda T, Abotchie $\mathrm{P}$, Mullen PD. Factors associated with patient and health care system delay in diagnosis and treatment for TB in sub-Saharan African countries with high burdens of TB and HIV. Trop Med Int Health. 2011; 16(4):394-411.

22. Bosanac EM, Parkinson RC, Hall DS. Geographic access to hospital care: a 30-minute travel time standard. Med Care. 1976; 14(7):616-24.



